\title{
NORM CONVERGENCE OF SECTORIAL OPERATORS ON VARYING HILBERT SPACES
}

\author{
Delio Mugnolo, Robin Nittka and Olaf Post
}

\begin{abstract}
Convergence of operators acting on a given Hilbert space is an old and well studied topic in operator theory. The idea of introducing a related notion for operators acting on varying spaces is natural. Many previous contributions to this subject consider either concrete examples of perturbations, or an abstract setting where weak or strong convergence of the resolvents is used. However, it seems that the first results on norm resolvent convergence in this direction have been obtained only recently, to the best of our knowledge. Here we consider sectorial operators on Hilbert spaces that depend on a parameter. We define a notion of convergence that generalises convergence of the resolvents in operator norm to the case when the operators act on different spaces. In addition, we show that this kind of convergence is compatible with the functional calculus of the operator and moreover implies convergence of the spectrum. Finally, we present examples for which this convergence can be checked, including convergence of coefficients of parabolic problems. Convergence of a manifold (roughly speaking consisting of thin tubes) towards the manifold's skeleton graph plays a prominent role, being our main application.
\end{abstract}

Mathematics subject classification (2010): 34B45, 35P05, 47D06.

Keywords and phrases: Diffusion equations on networks, approximation schemes, sectorial operators, norm convergence of operators in different Hilbert spaces, spectral convergence.

\section{REFERENCES}

[1] W. Arendt, M. Biegert, And A. F. M. Ter Elst, Diffusion determines the manifold, J. Reine Angew. Math. 667 (2012), 1-25.

[2] W. Arendt And M. Chovanec, Dirichlet regularity and degenerate diffusion, Trans. Amer. Math. Soc. 362 (2010), 5861-5878.

[3] M. Ahues, A. Largillier, And B. V. Limaye, Spectral computations for bounded operators, Applied Mathematics (Boca Raton), vol. 18, Chapman \& Hall/CRC, Boca Raton, FL, 2001.

[4] W. ARENDT, Semigroups and evolution equations: functional calculus, regularity and kernel estimates, Evolutionary equations. Vol. I, Handb. Differ. Equ., North-Holland, Amsterdam, 2004, pp. 185.

[5] S. Andres And M. Von Renesse, Particle approximation of the Wasserstein diffusion, Journal of Functional Analysis 258 (2009), 3879-3905.

[6] C. CACCIAPUOTI AND D. FInCO, Graph-like models for thin waveguides with Robin boundary conditions, Asymptot. Anal. 70 (2010), 199-230.

[7] G. M. Coclite, A. Favini, G. R. Goldstein, J. A. Goldstein, And S. Romanelli, Continuous dependence on the boundary conditions for the Wentzell Laplacian, Semigroup Forum 77 (2008), $101-108$.

[8] G. M. Coclite, G. R. Goldstein, And J. A. Goldstein, Stability estimates for parabolic problems with Wentzell boundary conditions, J. Differential Equations 245 (2008), 2595-2626.

[9] D. DANERS, Domain perturbation for linear and semi-linear boundary value problems, Handbook of differential equations: stationary partial differential equations. Vol. VI, Handb. Differ. Equ., Elsevier/North-Holland, Amsterdam, 2008, pp. 1-81.

[10] P. EXNER AND O. Post, Approximation of quantum graph vertex couplings by scaled Schrödinger operators on thin branched manifolds, J. Phys. A 42 (2009), 415305, 22. 
[11] U. R. FREIBERG AND M. R. LANCIA, Energy forms on conformal $C^{1}$-diffeomorphic images of the Sierpinski gasket, Mathematische Nachrichten 281 (2008), 337-349.

[12] D. GRIESER, Spectra of graph neighborhoods and scattering, Proc. Lond. Math. Soc. (3) 97 (2008), $718-752$.

[13] M. HAASE, The functional calculus for sectorial operators, Operator Theory: Advances and Applications, vol. 169, Birkhäuser Verlag, Basel, 2006.

[14] M. HinZ, Approximation of jump processes on fractals, Osaka J. Math 46 (2009), 141-171.

[15] K. ITO AND F. KAPPEL, Evolution equations and approximations, Series on Advances in Mathematics for Applied Sciences, vol. 61, World Scientific Publishing Co. Inc., River Edge, NJ, 2002.

[16] A. KASUE, Convergence of Riemannian manifolds and Laplace operators I, Ann. Inst. Fourier (Grenoble) 52 (2002), 1219-1257.

[17] A. KASUE, Convergence of Riemannian manifolds and Laplace operators II, Potential Anal. 24 (2006), 137-194.

[18] T. Kato, Perturbation theory for linear operators, Classics in Mathematics, Springer-Verlag, Berlin, 1995, Reprint of the 1980 edition.

[19] A. Kolesnikov, Mosco convergence of Dirichlet forms in infinite dimensions with changing reference measures, Journal of Functional Analysis 230 (2006), 382-418.

[20] S. M. Kozlov, O. A. OLEINIK, AND V. V. ZHIKOV, Homogenization of differential operators and integral functionals, Springer-Verlag, Berlin, 1994, Translated from the Russian by G. A. Yosifian [G. A. Iosif ${ }^{\prime}$ yan].

[21] K. KUWAE AND T. SHIOYA, Convergence of spectral structures: a functional analytic theory and its applications to spectral geometry, Comm. Anal. Geom. 11 (2003), 599-673.

[22] P. C. Kunstmann And L. WeIs, Maximal $L_{p}$-regularity for parabolic equations, Fourier multiplier theorems and $H^{\infty}$-functional calculus, Functional analytic methods for evolution equations, Lecture Notes in Math., vol. 1855, Springer, Berlin, 2004, pp. 65-311.

[23] T. A. MEL'NYK, Asymptotic analysis of a spectral problem in a periodic thick junction of type 3: 2: 1, Math. Meth. Appl. Sci. 23 (2000), 321-346.

[24] T. A. MEL'NYK, Hausdorff convergence and asymptotic estimates of the spectrum of a perturbed operator, Z. Anal. Anwendungen 20 (2001), 941-957.

[25] T. A. MEL'NYK, A scheme for studying the spectrum of a family of perturbed operators and its application to spectral problems in thick junctions, Nelinı̄̄n- Koliv. 6 (2003), 233-251.

[26] T. A. MEL'NYK, Homogenization of a boundary-value problem with a nonlinear boundary condition in a thick junction of type 3: 2: 1, Math. Meth. Appl. Sci. 31 (2008), 1005-1027.

[27] U. Mosco, Approximation of the solutions of some variational inequalities, Ann. Sc. Norm. Super. Pisa, Cl. Sci., III Serie 21 (1967), 373-394.

[28] U. Mosco And M. Vivaldi, Fractal reinforcement of elastic membranes, Archive for rational mechanics and analysis 194 (2009), 49-74.

[29] O. A. Olĕ̌nik, A. S. Shamaev, And G. A. Yosifian, Mathematical problems in elasticity and homogenization, Studies in Mathematics and its Applications, vol. 26, North-Holland Publishing Co., Amsterdam, 1992.

[30] E. M. Ouhabaz, Analysis of heat equations on domains, London Mathematical Society Monographs Series, vol. 31, Princeton University Press, Princeton, NJ, 2005.

[31] O. Post, Spectral convergence of quasi-one-dimensional spaces, Ann. Henri Poincaré 7 (2006), 933973.

[32] O. Post, Spectral analysis on graph-like spaces, Lecture Notes in Mathematics, Springer-Verlag, Berlin, 2011.

[33] S. E. Pastukhova And V. V. Zhikov, On the Trotter-Kato theorem in a variable space, Funktsional. Anal. i Prilozhen. 41 (2007), 22-29, 96.

[34] E. SÁncheZ-PALEncia, Nonhomogeneous media and vibration theory, Lecture Notes in Physics, vol. 127, Springer-Verlag, Berlin, 1980.

[35] P. Stollmann, A convergence theorem for Dirichlet forms with applications to boundary value problems with varying domains, Math. Z. 219 (1995), 275-287.

[36] G. Stolz and J. Weidmann, Approximation of isolated eigenvalues of general singular ordinary differential operators, Results Math. 28 (1995), 345-358.

[37] S. TEUfEL AND J. WACHSMUTH, Effective hamiltonians for constrained quantum systems, preprint arXiv:0907.0351, 2009. 
[38] J. Weidmann, Stetige Abhängigkeit der Eigenwerte und Eigenfunktionen elliptischer Differentialoperatoren vom Gebiet, Math. Scand. 54 (1984), 51-69. 